\title{
Cost Outcomes of Physical Therapist Providers Compared to Primary Care Providers in the Treatment of Musculoskeletal Patients in a Military Treatment Facility: A Retrospective Chart Review"
}

Troy E. McGill

Alaska Regional Hospital, troyemcgill@aol.com

Debra F. Stern

Nova Southeastern University, debras@nova.edu

Morey J. Kolber

Nova Southeastern University, kolber@nova.edu

John McGee

Marist College, John.mcgee@marist.edu

Follow this and additional works at: https://nsuworks.nova.edu/ijahsp

Part of the Diagnosis Commons, Musculoskeletal System Commons, Physical Therapy Commons, Physiotherapy Commons, Primary Care Commons, and the Radiology Commons

\section{Recommended Citation}

McGill TE, Stern DF, Kolber MJ, McGee J. Cost Outcomes of Physical Therapist Providers Compared to Primary Care Providers in the Treatment of Musculoskeletal Patients in a Military Treatment Facility: A Retrospective Chart Review". The Internet Journal of Allied Health Sciences and Practice. 2021 Jan 01;19(2), Article 4.

This Manuscript is brought to you for free and open access by the College of Health Care Sciences at NSUWorks. It has been accepted for inclusion in Internet Journal of Allied Health Sciences and Practice by an authorized editor of NSUWorks. For more information, please contact nsuworks@nova.edu. 


\title{
Cost Outcomes of Physical Therapist Providers Compared to Primary Care Providers in the Treatment of Musculoskeletal Patients in a Military Treatment Facility: A Retrospective Chart Review"
}

\begin{abstract}
Purpose: The purpose of this study was to compare the cost of physical therapist (PT) management of patients with musculoskeletal (MSK) disorders to management by traditional primary care managers (PCMs); medical doctors (MDs), doctors of osteopathic medicine (DOs), advanced registered nurse practitioners (ARNPs), and physician assistants (PAs). Methods: This is a retrospective study of electronic medical records using an exploratory, non-experimental, cross-sectional, and quantitative design method. The records of patients with MSK disorders were assessed at an Air Force military medical clinic for 18 months from January 2016 through June 2017. Results: PT management of MSK patients resulted in a significantly lower rate of imaging studies, NSAIDS, and cost of care when compared to MDs, DOs, PAs, or ARNPs. Patients with MSK disorders managed by PTs had no significant difference in return-to-work rate when compared to MDs, DOs, PAs, or ARNPs. Conclusions:The military model of physical therapy direct access care has been shown to be cost effective when compared to care provided by traditional primary care managers.
\end{abstract}

\section{Author Bio(s)}

Troy McGill, Lt Col, BSC, USAF (ret), PT, PhD, SCS, Dip MDT. Dr. McGill is a retired USAF Lt Col, He currently serves as the Director of Therapies and Health Management departments for Alaska Regional Hospital.

Debra F. Stern, PT, DPT, DBA. is the Director of Clinical Education in the Physical Therapy Department at Nova Southeastern University in the Dr. Pallavi Patel College of Health Care Science, Ft Lauderdale FL

Morey J. Kolber, PT, PhD, is a Professor, Department of Physical Therapy with Nova Southeastern University in the Dr. Pallavi Patel College of Health Care Science, Ft Lauderdale FL

John McGee, Lt Col, BSC, USAF (ret) PT, PhD, MBA, OCS, ATC, is an Assistant Professor in the Doctor of Physical Therapy Program at Marist College in Poughkeepsie, NY 


\title{
IIAHSP
}

\section{The Internet Joumnal of Allied Health Sciences and Practice \\ Dedicated to allied health professional practice and education}

Vol. 19 No. 2 ISSN 1540-580X

\section{Cost Outcomes of Physical Therapist Providers Compared to Primary Care Providers in the Treatment of Musculoskeletal Patients in a Military Treatment Facility: A Retrospective Chart Review}

\author{
Troy E. McGill1 \\ Debra F. Stern² \\ Morey J. Kolber² \\ John $\mathrm{McGee}^{3}$ \\ 1. Alaska Regional Hospital \\ 2. Nova Southeastern University \\ 3. Marist College \\ United States
}

\begin{abstract}
Purpose: The purpose of this study was to compare the cost of physical therapist (PT) management of patients with musculoskeletal (MSK) disorders to management by traditional primary care managers (PCMs); medical doctors (MDs), doctors of osteopathic medicine (DOs), advanced registered nurse practitioners (ARNPs), and physician assistants (PAs). Methods: This is a retrospective study of electronic medical records using an exploratory, non-experimental, cross-sectional, and quantitative design method. The records of patients with MSK disorders were assessed at an Air Force military medical clinic for 18 months from January 2016 through June 2017. Results: PT management of MSK patients resulted in a significantly lower rate of imaging studies, NSAIDS, and cost of care when compared to MDs, DOs, PAs, or ARNPs. Patients with MSK disorders managed by PTs had no significant difference in return-to-work rate when compared to MDs, DOs, PAs, or ARNPs. Conclusions:The military model of physical therapy direct access care has been shown to be cost effective when compared to care provided by traditional primary care managers.
\end{abstract}

Keywords: primary care physical therapy, physical therapy radiology, physical therapy pharmacology, physical therapy direct access 


\section{INTRODUCTION}

By 2030, an estimated total physician shortfall within the US of between 42,600 and 121,300 is expected. ${ }^{1}$ Of special interest is the predicted shortfall within primary care physicians of between 14,800 and 49,300 because these providers function as the gatekeeper for all other healthcare services. ${ }^{1}$ This shortage has opened the way for physician assistants (PAs) and advanced registered nurse practitioners (ARNPs) to fill the void by providing primary care medicine. ${ }^{2}$ These non-physician primary care managers (PCMs) have helped ease the access-to-care burden the U.S. health care system is experiencing along with providing safe, effective care at decreased cost. ${ }^{2}$ Even with these additional providers, there continues to be a significant shortage of PCMs. The Institute of Medicine (IOM) predicts that the shortage of U.S. PCMs will likely worsen as a result of the aging population, an increase demand for services because of the affordable care act, and decreased reimbursement for primary care providers. ${ }^{1,2,3}$ Advances in PT education, training, and practice capabilities, coupled with legislative changes, have given PTs the opportunity to prove themselves to be safe, effective, direct access providers. ${ }^{4}$ PTs offer a viable solution for substantially improving access to care for certain well-defined populations. ${ }^{4,5,6,7,8}$

Physical therapy (PT) has advanced along a continuum of increased responsibility from a physician-directed, prescriptive occupation to a profession where autonomous practice is more common. 7,8 Department of Defense (DoD) and civilian PTs who practice in military treatment facilities (MTFs) function as PCMs for MSK patients. They are covered by federal law which grants practice privileges that include ordering diagnostic imaging and laboratory studies, prescribing medications, performing joint injections, and restricting work-related activities. ${ }^{9}$ All military services require advanced training through various military education programs that ensure safe, standardized care when patients access a PT through direct access. ${ }^{9}$

Physical therapists are educated at the Doctorate level and are ready to serve as MSK PCMs. Current U.S. DPT programs provide the didactic preparation sufficient for independent practice. However, specific instruction or practice within an environment that allows writing prescriptions and ordering laboratory and imaging studies is not part of most clinical internships, unless the student PT has the opportunity to intern in a MTF. ${ }^{4}$ Department of Defense PTs, who serve as MSK PCMs, function as part of a primary care team. If patients are beyond the scope of PT care, then the PT would refer to a provider who is better equipped to determine the plan of care. All medical providers, including traditional PCMs, understand their scope of practice and its limitations and refer when appropriate. In addition, PTs utilized as a MSK PCM would complement other physician and non-physician PCMs who focus often on non-MSK issues.

This study compared cost of PT management of patients with MSK disorders to management by traditional PCMs; medical doctors (MDs), doctors of osteopathic medicine (DOs), ARNPs, and PAs.

\section{METHODS}

A retrospective review of electronic medical records (EMRs) using an exploratory, non-experimental, cross-sectional, quantitative design was conducted. All patients with MSK complaints over the age of 18 who were eligible to receive care at a MTF were given the opportunity to be evaluated by a USAF PT through direct-access, same-day care. The ability to examine effectiveness of PTs in a direct access setting could only occur in a DoD MTF because of the breadth of practice privileges that DoD PTs possess. This study is designed to examine possible differences in cost of care for patients with MSK pathology between the five PCM groups in the DoD: 1) PTs, 2) MDs, 3) DOs, 4) PAs, and (5) ARNPs.

\section{Study Participants}

The patient sample consisted of 8,053 patients with MSK disorders who sought care at a military medical clinic during the 18month period from Jan 2016 to June 2017. In most DoD facilities, PTs serve as the MSK PCM and ARNPs, PAs, MDs and DOs provide MSK care but primarily provide non-MSK care. All patients over the age of 18 with MSK conditions were eligible for the EHR review. There are several ways to access PT in the MTF.

1. Direct self-reporting.

2. Indirect self-reporting: Patients call the central appointment desk and their MSK condition is triaged by an appointment clerk using the algorithm shown in Table 1.

3. Patient sees their PCM and is referred to PT. 
Table 1. Appointment Algorithm

\begin{tabular}{|l|c|l|}
\hline \multicolumn{1}{|c|}{ Complaint } & \multicolumn{1}{c|}{ Action } \\
\hline $\begin{array}{l}\text { If pain in a muscle or joint with no } \\
\text { deformity to the limb }\end{array}$ & Then & Refer to PT direct access clinic \\
\hline $\begin{array}{l}\text { If pain from a motor vehicle accident } \\
\text { (MVA) and patient was seen in the ER }\end{array}$ & Then & Refer to PT direct access clinic \\
\hline $\begin{array}{l}\text { If pain in a muscle or joint in an } \\
\text { adolescent 17 or under }\end{array}$ & Then & Refer to Pediatric Clinic \\
\hline If pain with bone deformity & Then & Refer to Emergency Department \\
\hline $\begin{array}{l}\text { If the patient has a strain, sprain, or } \\
\text { "pulled" muscle }\end{array}$ & Then & Refer to PT direct access clinic \\
\hline
\end{tabular}

\section{Data Analysis}

Collection of patient data was processed through Air Force Medical Service Analytics by means of retrieval of patient information from the electronic medical records systems (Table 2). Demographic data for each patient included the following: age in years, gender, ethnicity, and military rank. Patient characteristic variables included tobacco use and pain. Coded indicator variables were used to classify each patient's PCM group and MSK site of injury. Dependent variables included all imaging ordered, NSAID prescription rate, and the number of patient visits until return to work (RTW). All data was analyzed with IBM ${ }^{\odot}$ SPSS ${ }^{\circledR}$ Statistics Version 22.

Table 2. Frequency and Percentage Summaries of Categorical Measured Demographic Data and Data of Independent Variables. The dependent variables include frequency of imaging, prescription rate of NSAIDS, number of visits until RTW, cost of visits dependent on PCM type.

\begin{tabular}{|l|c|c|}
\hline Age Category (years) & Frequency & Percent \\
\hline $18-24$ & 1449 & 18.0 \\
\hline $25-34$ & 2662 & 33.1 \\
\hline $35-44$ & 2064 & 25.6 \\
\hline $45-64$ & 1684 & 20.9 \\
\hline $65+$ & 194 & 2.4 \\
\hline & & \\
\hline Gender & & 65.7 \\
\hline Male & 5290 & 34.3 \\
\hline Female & 2763 & \\
\hline & & Percent \\
\hline Ethnicity & Frequency & 4.3 \\
\hline Black & 343 & 33.6 \\
\hline White & 2707 & 0.2 \\
\hline Hispanic & 17 & 2.0 \\
\hline Asian & 165 & 21.2 \\
\hline Other non-White & 1709 & \\
\hline & & 43.1 \\
\hline Patient Rank & & 50.2 \\
\hline 1.0 Junior enlisted & 3467 & 2.8 \\
\hline 2.0 Senior enlisted & 4039 & 3.9 \\
\hline 3.0 Junior officer & 223 & 0.1 \\
\hline 4.0 Senior officer & 317 & \\
\hline Missing & 7 & 6.5 \\
\hline Tobacco Use & & \\
\hline Yes & 523 & \\
\hline No & 5544 & \\
\hline Pain (0-10 VAS) & \\
\hline
\end{tabular}




\begin{tabular}{|l|c|c|}
\hline \multicolumn{1}{|c|}{ 0 No pain } & 831 & 10.3 \\
\hline $1-6$ & 396 & 5.0 \\
\hline $6-9$ & 390 & 4.8 \\
\hline 10 Intense pain & 44 & 0.5 \\
\hline \#MULTIVALUE 1-8 & 5926 & 73.6 \\
\hline Injury Location & & \\
\hline Spine & 111 & 1.4 \\
\hline Shoulder & 849 & 10.5 \\
\hline Knee & 1549 & 19.2 \\
\hline Ankle & 601 & 7.5 \\
\hline MSK other multiple joint & 4943 & 61.4 \\
\hline & & \\
\hline Specialty provider & & \\
\hline PT & 1781 & 22.1 \\
\hline MD & 3582 & 44.5 \\
\hline DO & 145 & 1.8 \\
\hline PA & 1962 & 24.4 \\
\hline ARNP & 583 & 7.2 \\
\hline
\end{tabular}

\section{RESULTS}

Imaging

A level of significance of 0.05 was used for the ANOVA. Imaging values for patients during the episode of care ranged from 0 (if no imaging was ordered) to 56 images ordered, with a mean of 3.57 images ordered (SD $=2.96)$. The ranges of values of the mean diagnostic imaging cost with a $p<0.02$ for initial MSK injury encounter for the 8,053 patients was from $\$ 59.00$ to $\$ 116.33$, while the mean was $\$ 64.50$ ( $S D=\$ 11.61$ ). If a radiology study was not ordered, no radiology cost was associated with the visit. One-way ANOVA and Tukey post hoc testing revealed that for plain-film imaging, PAs and ARNPs functioning as PCMs ordered significantly more imaging studies of their MSK patients than PTs, MDs or DOs functioning as PCMs, by mean differences of -0.10 , .42 and 0.54 respectively, with a $p<0.336$ with PTs ordering the fewest imaging studies. Radiology usage for all PCM groups was higher than that of PTs serving as PCMs.

\section{Medications (NSAIDs)}

Cost of prescribed anti-inflammatory medications ranged in values from $\$ 0.00$ cost (when no medication was prescribed) to $\$ 37.00$. The mean was $\$ 22.00$ ( $S D=\$ 30.01$ ). One-way ANOVA and Tukey post hoc testing revealed that for medication use, PTs acting as PCMs for MSK patients prescribed significantly less medication (NSAIDs) than MDs, DOs, PAs, and ARNPs acting as PCMs for MSK patients, by mean differences of $-16.1133,-9.9057,-13.8669$, and -12.6703 , respectively, with a $p<.001$. The PTs in this study prescribed significantly fewer NSAIDs than their MD, DO, PA and ARNP counterparts. Overall, patient acuity was similar in all five groups, with PTs, on average, seeing patients with a pain rating of five out of 10 , and the other PCMs, on average, seeing patients with a pain rating of 4 out of 10 .

\section{Cost of Care}

Number of visits, as measured by individual patient encounters, ranged from one visit to 10 visits, with a mean of 1.23 visits (SD = 0.81). The range of cost per episode care, based on the number of patient visits, ranged from $\$ 100$ to $\$ 2,000$, and the mean was $\$ 222.30$ (SD = \$163.26). For costs of visits, MSK patients seeing PTs in the role of PCM had a significantly lower cost of care per visit than MSK patients seeing MDs, DOs, PAs, and ARNPs in the PCM role, by mean differences of $\$-106.56, \$-51.15, \$-100.62$, and $\$-99.53$ respectively with a $p<.001$. On average, the cost of military medical care provided to patients with MSK disorders by PTs acting as the PCM was half that of the cost of care provided to patients with MSK disorders by MDs, DOs, PAs, and ARNPs acting as the PCM.

\section{Return to Work}

Patients had a significantly greater number of visits when MDs served as PCMs than when PT served as the PCM, with a p<.000. However, no significant differences were reported in number of visits among all PCM types when hierarchical linear regression controlled for demographics and for patient characteristics and comorbidities. Yet, prior to controlling for effects of individual variables, post hoc results using Tukey's Statistics of Differences revealed that when patients with MSK conditions saw MDs as their PCM, they had a significantly more visits before discharge than patients with MSK conditions who saw PTs as their PCM, by 
a mean difference of 0.17 . Number of visits and then discharge were used as return-to-work criteria. The military electronic health record (AHLTA) did not allow access to time-based data that would allow analysis of time from initial evaluation to discharge. Limitation of data to number of visits only did allow for cost per visit to be calculated. Consequently, it was not possible to determine if PTs were able to return patients to work quicker than other non-PT PCMs.

\section{DISCUSSION}

The question of patient safety is paramount when a medical provider functions as the patients' healthcare entry point provider. Are PTs safe, are their differential diagnostic skills sufficient to determine when to refer or when to continue the assessment and progress to offering treatment? In a recent retrospective descriptive study completed at a university health center, no unidentified cases of serious medical pathology or adverse events were reported as a result of direct access to PT. ${ }^{10}$ The University of Colorado at Boulder instituted a direct access musculoskeletal injury clinic in 2000. A retrospective analysis performed on patient visits to Wardenburg Student Health Center from January 1, 2001 to December 31, 2011. Descriptive statistics were analyzed for the number of new patients examined with and without a referral, documented patient adverse events, and any disciplinary or legal action against a physical therapist. During the 10-year data collection period, 12,976 patients accessed PT without a referral. It was concluded that patients managed through direct access are at minimal to no risk for negligent care when evaluated and treated by PTs in a university student health center setting. ${ }^{10}$

It was reported that patient access to care improved in this direct access PT model, compared to access to traditional PCMs. In a recent survey, patients were asked about multiple issues pertaining to healthcare. Regarding access, it was reported that the average wait time to see a PCM in 15 metropolitan areas was almost three-weeks. ${ }^{4}$ It was noted by a physician who helped conduct the survey that "most primary care doctors have their available timeslots pre-booked with chronic care patients who take longer to see: 20-30 minutes for a routine follow-up versus 10-15 minutes for straightforward cases like a sore throat or twisted ankle."4

Currently, traditional PCMs have increased liability when compared to PTs. A 2010 study reported that $91 \%$ of physicians practice defensive medicine, ordering more tests and procedures than necessary to help protect themselves from lawsuits. ${ }^{11}$ The study asked physicians to agree or disagree with two questions in order to rate the physicians' level of agreement: 1) Doctors order more tests and procedures than patients need for [legal] protection; and 2) without tort reform, unnecessary diagnostic testing will not decrease. An overwhelming $91 \%$ of physicians agreed with both statements. ${ }^{11}$ Advanced registered nurse practitioners and PAs who practice as front-line providers face the same litigation pressures that physicians face. Military PTs who serve as front-line providers are much less likely to face lawsuits because of the protection provided by the 1940 Federal Torts Claims Act. ${ }^{12,13}$ Physical therapy has made great strides in increasing education standards to meet the demands of more specialized care. Entry level degree standards have increased from the initial requirement in the 1960s of a certificate level, to a bachelor's degree, then to a master's degree, and now to a clinical Doctor of Physical Therapy degree for all U.S. accredited programs. ${ }^{14,15}$ With the expansion of entry-level curricular content to include pharmacology, radiology, and differential diagnosis, the ability of a PT to serve as an entry-level professional for patients in certain areas is a logical step, although, as in the military, there may need to be some additional clinical training. As of January 1, 2015, all 50 states as well as the District of Columbia and the U.S. Virgin Islands allow patients to seek some level of treatment from a licensed PT without a prescription or referral from a physician. ${ }^{16}$ In very limited civilian practices, PTs are evaluating patients as direct access providers. ${ }^{17}$ Physical therapist have proven to be cost effective, safe providers who have increased accessed and streamlined recovery. ${ }^{17,18,20}$

The results of this study demonstrated the prudent use of imaging by two PT's in a single military treatment facility with approximately 1,700 patients evaluated through direct access over a period of 18 months. Allowing PTs to use radiology exams when clinical indicators dictate a need could help reduce healthcare costs without negatively effecting quality care and outcomes. Use of imaging CPGs and a thorough MSK examination by PTs to clarify patients' clinical presentations will help identify patients who evidence-based medicine would indicate need further evaluation.20,21 This study was unable to assess electronic healthcare documentation data to determine if use of radiology CPGs guided decision making of these five PCM groups. It was not possible to assess each individual record for PCM imaging or CPG free-text documentation, as there was no check box that recorded this information. There are multiple studies that have examined the efficacy of the military model of PT in which the ability to order radiology has been common since the early 1970s. These studies have demonstrated that military PTs order imaging prudently and have not negatively affected patient safety. ${ }^{21,22}$ The civilian sector is recognizing that PTs have the clinical skill sets required to competently acquire radiology ordering privileges. Kaiser Permanente Northern California developed specific radiology competencies for PTs who practice in their healthcare system. ${ }^{17,23,24}$ Kaiser has moved physical therapy to a primary care, frontline provider for MSK conditions, with $\mathrm{x}$-ray privileges granted once the specific clinical competencies are satisfied. ${ }^{23}$ Kaiser, like the military, developed specific pathways for PTs to provide primary care by using PTs in a way that would ensure full use of their clinical capacities. Kaiser, like the military, realized that PTs had an unused skill set that could be used to provide care to certain patient types. 
In the U.S., only the military grants PTs practice privileges to prescribe medications - specifically NSAIDs. However, since 2006, PTs in the United Kingdom (UK) were able to prescribe medication as supplementary prescribers, requiring a physician's cosignature on their prescriptions. ${ }^{25}$ Physical therapists in the UK have been using medicines for injection therapy since the early 1990s under physician supervision the way that PAs have been doing in the United States. Since 2000, local anesthetics and corticosteroids have been used extensively by the estimated 3,000 PTs in the UK. ${ }^{25}$ Supplementary prescribing is also used in a broad range of community and acute settings. Physical therapists in the UK use these practice privileges within a range of relevant medicines in clinical areas including musculoskeletal treatment, pain management, neurological care, respiratory treatment, emergency care, women's health, pediatrics, and geriatric medicine. ${ }^{25}$ These medications are not limited to a certain class such as NSAIDs, but are specific to the type of practice setting and covers all types of medication, including narcotics. ${ }^{25}$ When this initiative yielded positive results and no reported adverse patient complaints, PTs in the UK were granted independent prescribing privileges in 2012.25 The Chartered Society of Physiotherapy noted that it "would like to see the same changes take place in the United States of America," but said it is "doubtful that the American medical establishment would be as supportive of physical therapists as the British physicians seem to be; the fear of lower physician salaries would draw political opposition from the American Medical Association." 25

This study was conducted at one Air Force MTF, examining the practice patterns of two PTs, two PAs, one ARNP, two MDs, and three DOs. Generalization to other practice settings would be limited. However, results support the value of additional research in practice settings where cost and patient outcomes of traditional PCMs could be compared to PTs serving in the same role. Wisconsin is the first state to allow PTs to order imaging studies - an effort that should be assessed to hopefully lend support for similar measures to be pushed to other states.26,27 The American Physical Therapy Association is supportive of efforts - such as granting imaging privileges - to assist with autonomous PT practice. ${ }^{27}$ Additionally, the ability to determine whether providers documented use of any form of radiology clinical practice guidelines prior to ordering $\mathrm{x}$-rays was not available. The examination if usage frequency and documentation of current radiology clinical practice guidelines could be an area of future reach.

Finally, there is no evidence to support the conclusion that PTs provide less expensive care in the long-term within the current US healthcare system. The possibility exists that as payers and patients acclimate to PTs serving as entry point providers, cost may mirror that of other non-PT PCMs. Even if this is the case, it could be argued that patients who see PTs as front-line providers would receive the most competent, non-operative care for MSK conditions. Overall, the results of this study are preliminary with the suggestion of a follow-up study that risk adjusted patients based on comorbidities. This study provided the unique opportunity to examine PTs functioning in the role of MSK PCM compared to traditional PCMs functioning in the same role.

\section{References}

1. Association of American Medical Colleges, 2018. The Complexities of Physician Supply and Demand: projections from 2016 to 2030. 2018 update. IHS marketing Ltd. Washington D.C.

2. Association of American Medical Colleges, 2008. The Complexities of Physician Supply and Demand: Projections through 2025. 2008b. IHS Marketing Ltd. Washington D.C.

3. Army-Baylor DPT Graduate School. 2018. Retrieved from: http://www.baylor.edu/graduate/pt/index. php?id =28285.

4. Duke University, Mission statement, 2016. Retrieved from: https://dpt.duhs.duke.edu/.

5. Deyle GD. Direct access physical therapy and diagnostic responsibility: The risk-to-benefit ratio. J Orthop Sports Phys Ther. 2006;36(9):632-634.

6. McCallum CA, Di Angelis T. Direct access: Factors that affect physical therapist practice in the state of Ohio. Phys Ther. 2012;92(5):688-706.

7. Mintken P, Pascoe C, Barsch S, et al. Direct Access to Physical Therapy Services Is Safe in a University Student Health Center Setting. J Allied Health. 2015;44. 164-168.

8. Air Force Instruction. Clinical performance improvement. Title 10, U.S.C. Chapter 55, Sections 1094 and 1102. April 2014.

9. New Choice Health, Your healthcare marketplace (2016, Jan). Retrieved from https://www.newchoicehealth.com/XRay/medication-Cost

10. The Physician Foundation, 2017. Patient survey report for the physician foundation. Regina Corso Consulting, New York, NY.

11. Crane M. 2010. New Study Finds $91 \%$ of Physicians Practice Defensive Medicine. Medscape. June 28, 2010. Retrieved from https://www.medscape.com/viewarticle/724254

12. U.S. House of Representatives, 2018. Federal Tort Claims Act (2018).

13. American Physical Therapy Association. Direct access to physical therapy services: Overview. 2015.

14. Dill M., Salsberg E. HHS in the 21st century: Charting a new course for a healthier America. 2008a. 
15. American Physical Therapy Association. Today's physical therapist: A comprehensive review of a 21 st-century health care profession. 2011.

16. Shoemaker MJ. Direct consumer access to physical therapy in Michigan: Challenges to policy adoption. Phys Ther. 2012;92(2):236-250.

17. American Physical Therapy Association. Indiana enacts direct access: Direct PT evaluation now in all 50 states + DC. PT in Motion News. 2013.

18. Commission on Accreditation in Physical Therapy Education. Evaluative criteria PT programs. 2016. Retrieved from: http://www.capte

19. Childs JD, Whitman JM, Pugia ML, et al. Knowledge in managing musculoskeletal conditions and educational preparation of physical therapists in the uniformed services. Mil Med. 2007;172(4):440-445.

20. Moore JH, Goss DL, Baxter RE, et al. Clinical diagnostic accuracy and magnetic resonance imaging of patients referred by physical therapists, orthopedic surgeons, and orthopedic providers. J Orthop Sports Phys Ther. 2005;35(2):67-71.

21. McGill T. Effectiveness of physical therapists serving as primary care musculoskeletal providers as compared to family practice providers in a deployed combat location: A retrospective medical chart review. Mil Med. 2013;178(10):11151120.

22. Tischner CJ. Kaiser Permanente moves forward with physical therapists in primary care. Orthop Pract. 1998; 10:19.

23. Department of Health. Consultation on Proposals to Introduce Independent Prescribing by Physiotherapists.' London: HMSO; 2011.

24. Wisconsin State Legislature. 2015-2016 Legislature 2015 Assembly Bill 549 (November 24, 2015).

25. United Kingdom's Chartered Society of Physiotherapy. 2016. http://www.csp.org.uk/. Accessed September 7, 2015.

26. Dominiczak L, Schick K. Advancing scope of practice - Physical Therapists ordering X-rays in Wisconsin. 2016 Federation of State Boards of Physical Therapy annual meeting. www.qa.fsbpt.org/.../ Forum_Winter2016_WisconsinPTXrays.pdf. Accessed June 5, 2016.

27. American Physical Therapy Association. The task force on strategic plan to achieve vision 2020; a vision statement for physical therapy 2020. Working operational definitions of elements of vision 2020. 2008. http://www.apta.org/Vision2020/. Accessed July 5, 2016. 